\title{
The ridge through colored glass
}

\author{
Raju Venugopalan \\ Physics Department, Brookhaven National Laboratory, Upton, NY 11973, USA
}

\begin{abstract}
We briefly outline the role of gluon saturation, and the interplay between initial and final state effects, in generating the ridge observed in high multiplicity proton-proton and proton-nucleus collisions.
\end{abstract}

\section{Introduction: High multiplicity events and gluon saturation}

The striking data on azimuthally collimated long range rapidity correlations in proton-nucleus $(\mathrm{p}+\mathrm{A})$ collisions presented at Quark Matter 2014 were one of the highlights of the conference. There is a strong viewpoint that these results present compelling evidence for collective flow of the produced matter, as opposed to being primarily an initial state effect. We shall examine this viewpoint closely, if briefly, in this note. We shall also recapitulate the pre-Quark Matter studies that argued that key features of the data can be understood as an initial state phenomenon. Since Quark Matter, several papers have appeared that have an initial state perspective. These too will be addressed within space constraints.

Regardless of whether collective flow drives the effect, or not, the dynamics of the initial state holds the key to understanding the phenomenon. By this we mean specifically the quark and gluon configurations in the proton and the nucleus that are triggered in high multiplicity events, as well as the preequilibrium dynamics that may occur prior to the time when hydrodynamics may be relevant. Consider proton-proton $(\mathrm{p}+\mathrm{p})$ collisions where a small but distinct ridge is seen in high multiplicity events. The highest multiplicity events triggered corresponded to charged particle multiplicities $N_{\text {ch. }} \geq 110$ particles in nearly 5 units of rapidity; these are rare approximately one in a million events.

It is natural to ask what the spatial structure is of the quark-gluon configurations in the proton that generate such large numbers of hadrons. The guidance from the typical events studied in HERA inclusive deeply inelastic scattering (DIS) data is that the gluon momentum distribution behaves as $x d N / d x \propto 1 / x^{\lambda\left(Q^{2}\right)}$. The $\lambda\left(Q^{2}\right)$ extracted from data has an interesting structure [1]; it is nearly constant with $\lambda\left(Q^{2}\right) \approx 0.08$ until $Q^{2}=1 \mathrm{GeV}^{2}$, but rises subsequently approximately as $\ln \left(Q^{2}\right)$ with $\lambda\left(Q^{2}=10 \mathrm{GeV}^{2}\right) \sim 0.25$. Working in a frame where the rapidity evolution of gluons from the beam rapidity down to $y=0$ is in the wavefunction of one of the protons, one finds in the rapidity interval $0 \leq y \leq 2.4$ that $N\left(Q^{2}=2 \mathrm{GeV}^{2}\right) \sim 6-7$ gluons. This is obtained by integrating the gluon distribution over the corresponding $x$ range $\left(x_{\min .}, x_{\text {max. }}\right) \equiv\left(2 \cdot 10^{-4}, 2.2 \cdot 10^{-3}\right)$ with $\lambda\left(Q^{2}=2 \mathrm{GeV}^{2}\right)=0.14$. If all the gluons in the collision are released, one obtains about 13 gluons in nearly 5 units of rapidity, a far cry (assuming parton-hadron duality) from the $>110$ hadrons measured in the high multiplicity $\mathrm{p}+\mathrm{p}$ events. To obtain numbers that are in the ball park, one must have the effective value of $\lambda\left(Q^{2}=2 \mathrm{GeV}^{2}\right) \approx 0.4$, a very rapid rise of the gluon distribution indeed!

If there is a very rapid rise of the gluon distribution, when does the parton model picture of individual partons break down ? A simple estimate is obtained if we assume that each of the $O\left(10^{2}\right)$ gluons is of radius $2 / Q$, and one requires that they do not overlap. The only way to ensure this is to increase the gluon radius of the proton; for $N_{\text {ch. }} \sim 100$, our estimate would give $\sim 3 \mathrm{fm}$ ! The Hanbury-Brown-Twiss (HBT) radii (typically larger than the initial proton size) 
measured in $\mathrm{p}+\mathrm{p}$ collisions at the LHC, for comparable multiplicities are smaller than 2 Fermi [2]. If on the other hand one allows gluon modes with $k_{\perp} \leq Q_{S}$, where $Q_{S}$ is the saturation scale, to be maximally occupied with occupancies $\sim 1 / \alpha_{S}$, the gluon radius of the proton can be smaller. The number of produced gluons can be computed numerically in the CGC framework [3] and one obtains [4] $d N_{g}^{\text {prot. }} / d \eta \approx \frac{1.1 C_{F}}{2 \pi^{2}} \frac{S_{\perp} Q_{S \text {,prot. }}^{2}}{\alpha_{S}}$. Here $C_{F}=4 / 3$ and $S_{\perp}=\pi R_{\text {prot. }}^{2}$; for $R_{\text {prot. }}=0.8 \mathrm{fm}$ and $\alpha_{S}=0.3$, one obtains $d N_{g}^{\text {prot. }} / d \eta \sim 110$ for $Q_{S \text {,prot. }}^{2}=2 \mathrm{GeV}^{2}$.

There are several aspects of this back of the envelope estimate that are worthy of note. Firstly, at the price of a reasonable semi-hard scale, by allowing for occupancies $1 / \alpha_{S}$, one is able to accommodate the high multiplicities generated in the proton-proton collisions without requiring proton radii inconsistent with HBT analyses. Secondly, the saturation scale of $Q_{S \text {,prot. }}^{2}=2 \mathrm{GeV}^{2}$, while not apparently very large, is in fact quite large for a proton. To put this in context, saturation model fits to HERA inclusive and diffractive data give $Q_{S}^{2} \sim 0.5 \mathrm{GeV}^{2}$ at the center of the proton [5]. The additional factor of 4 in $Q_{S}^{2}$ obtained in rare, triggered LHC events is only otherwise attained in a multi-TeV center-of-mass e+p DIS collision. Finally, the approximate boost invariance of two particle correlations arises naturally in the Color Glass Condensate (CGC) framework that describes the properties of saturated gluons.

These considerations suggest that regardless of whether initial state or final state dynamics dominates, the gluons responsible for this dynamics exist in high occupied states characterized by a semi-hard dynamical saturation scale. If one accepts this, it then follows immediately that the highly occupied modes with $k_{T} \ll Q_{S}$ must experience some sort of collective dynamics; the open question is whether this collective dynamics is best described by (nearly ideal) hydrodynamics. Conversely, the dynamics of modes with $k_{T} \geq Q_{S}$ is not collective dynamics, but is nevertheless non-trivial dynamics described by the CGC-at large $k_{T}$ is should match on to perturbative QCD. We will next address what this initial state CGC dynamics is, and outline some of the open issues.

\section{Initial state descriptions of the ridge}

In a perturbative QCD framework, which should be valid for $k_{T} \geq Q_{S}$, there are two possibilities for generating two particle correlations. One of these corresponds to two gluons produced from the same ladder, while the other corresponds to two gluons produced from two ladders that share the momentum and color flow. The former is dubbed the "mini-jet" contribution, while the latter are the "Glasma graph" contribution. Both of these contributions should be there in any first principles QCD derivation; the only question is what their relative magnitude is. It was observed that if the occupancy of gluons in the hadron wavefunctions is large (as is the case for most gluons, since the median $k_{T} \sim$ $Q_{S}$ ), the contribution from the Glasma graphs becomes large, and is competitive with the mini-jet contribution [6].

For $\mathrm{p}+\mathrm{p}$ collisions, this two component description works extremely well. While there is a model dependence coming from the choice of the saturation scales at the beam rapidity, the evolution of these saturation scales with rapidity, and the relative magnitude of glasma graphs versus mini-jet are an outcome of the model, not to mention the detailed description of the matrix of transverse momenta $\left(p_{T}^{\text {trig. }}, p_{T}^{\text {assoc. }}\right)$. It is also important to note that the collimated glasma contributions are $1 / N_{c}^{2}$ relative to the leading uncollimated background; the latter scales as the multiplicity. The fact that the uncollimated background is large suggests that collimated contributions that are leading in $N_{c}$, while feasible in principle, are in practice small. In other words, the ratio of the collimated signal to the uncollimated background in $\mathrm{p}+\mathrm{p}$ collisions, has a magnitude that is approximately $1 / N_{c}^{2}$.

In $\mathrm{p}+\mathrm{A}$ collisions, the same framework describes the two particle correlation data semi-quantitatively [7]. In detail, however, one sees that the odd $v_{3}$ harmonic is significant. The simplified Glasma graph two gluon correlator we considered previously is symmetric under the exchange $C\left(\mathbf{p}_{\mathbf{T}}^{\text {trig. }}, \mathbf{p}_{\mathbf{T}}^{\text {assoc. }}\right) \rightarrow \mathbf{C}\left(\mathbf{p}_{\mathbf{T}}^{\text {trig. }},-\mathbf{p}_{\mathbf{T}}^{\text {assoc. }}\right)$, and therefore $v_{3}$ is zero. One contribution we had ignored previously is the interference between the mini-jet and glasma graphs that should be there in a complete computation. This contribution is zero when averaged over the direction of the impact parameter vector. If one assumes that the other particles in the event form a net $\mathbf{b}$ vector, as we shall discuss shortly, it is conceivable that this conclusion may be modified. Another potential source of $v_{3}$ are the power suppressed contributions (parametrically of order $Q_{S} / k_{T}$ ) that correspond to coherent multiple-scattering contributions. For large $k_{T}$ these are small, but they can be large for momenta smaller than the saturation scale. These were not included previously because a) our attention was focused on collimated contributions at large $k_{T}$, and b) they have to be computed numerically on the lattice. It is known however that with these coherent multiple scattering contributions $C\left(\mathbf{p}_{\mathbf{T}}^{\text {trig. }}, \mathbf{p}_{\mathbf{T}}^{\text {assoc. }}\right) \neq \mathbf{C}\left(\mathbf{p}_{\mathbf{T}}^{\text {trig. }},-\mathbf{p}_{\mathbf{T}}^{\text {assoc. }}\right)$, leading to a finite $v_{3}$ contribution from the Glasma graphs alone [8]. There should 
also be such coherent multiple scattering corrections to mini-jet production; both of these contributions are under investigation.

There have been a number of recent papers that have investigated initial state contributions to the observed azimuthal anisotropies in p+A collisions. One such work, by Gyulassy et al. [9], has as its building block the GunionBertsch $2 \rightarrow 3$ graph, which in high energy kinematics was first considered by Fadin and Lipatov. Unlike us though, they argue there is a leading $N_{c}$ contribution, which comes from the two correlated gluons having a net momentum transfer that is balanced by the other $n-2$ gluons in the event. This would be equivalent to the two gluons being corrrelated respective to an impact parameter direction formed by the other gluons. In our language, this could correspond to a non-eikonal contribution; the momenta of the two gluons are not conserved within the two gluon sub-graph. Alternately, this could be a geometric correlation from the possibility that the two gluon ladders could be correlated simply because the valence degrees of freedom generating these arise from the same wavefunction. (See for instance, [10].) The power counting for these contributions needs to be quantified more clearly relative to the glasma graphs. If geometric nucleon correlations play a big role, how does one explain the ridge in $p+p$ for instance, or the large uncollimated background?

Another recent initial state model assumes that there is a net color field direction in the nucleus experienced by partons in the incoming proton, event-by-event [11]. This is equivalent in our approach to assuming that the $Q_{S}^{2}$ kick in a single event is direction dependent. The correlation of particle production with this radial direction then produces the even and odd moments. There is an additional parameter in this approach corresponding to the degree of polarization of the color field. In practice, this variable is found to be quite small; which again, is consistent with having a large uncollimated background. We also note that a recent paper [12] studying the $v_{n}$ moments generated from multi-gluon emission from a cut pomeron obtains nearly identical $v_{n}\{m\}$ moments for $m \geq 4$; the implications of these need to be better understood.

Though there are a number of interesting ideas that argue for an initial state interpretation, there is at present no coherent framework to evaluate their relative contributions systematically, and to compare these to the data. One expects progress in this direction in the near future.

\section{Collectivity in small systems}

At Quark Matter, there was an emerging consensus that collectivity is seen in high multiplicity $\mathrm{p}+\mathrm{Pb}$ collisions. This was nicely summarized in the plenary presentation by Granier de Cassagnac [13], who adduced a number of pieces of evidence in this direction. We shall address each of these here.

- Strong $v_{2}$ from multiparticle correlations. The observation that $v_{2}\{4\} \approx v_{2}\{6\} \approx v_{2}\{8\}$ is indeed strongly suggestive of some form of collectivity. However, one should note that the integrated $v_{2}$ is dominated by low $k_{T}<Q_{S}$ modes, where coherent multiple scattering is important. While coherence is "collective", it is not necessarily so in a hydrodynamical sense.

- Similar mass ordering in $\mathrm{p}+\mathrm{A}$ and $\mathrm{A}+\mathrm{A}$. Mass ordering is natural in hydrodynamical models. However, the mass splitting of the $\left\langle p_{T}\right\rangle$ of hadrons is seen not just in high multiplicity events but also in minimum bias events with very few particles. This leaves open the possibility that the mass ordering may reflect universal hadronization patterns.

- $v_{2}$ depending on $\eta$ in $\mathrm{p}+\mathrm{Pb}$. The $\eta$ dependence of $v_{2}$ perhaps reflects more the underlying stringy picture of multiparticle production that is an input into hydrodynamics rather than being a feature of hydrodynamics per se. The $\eta$ dependence of two particle correlations is also reproduced in initial state scenarios.

- Same $v_{3}$ versus multiplicities in $\mathrm{p}+\mathrm{A}$ and $\mathrm{A}+\mathrm{A}$. While this observation is extremely interesting, it is unclear what it has to do with hydrodynamics. A counter example is provided by the IP-Glasma+MUSIC hydrodynamic model, where $v_{3}$ 's are significantly different in $\mathrm{p}+\mathrm{Pb}$ and $\mathrm{Pb}+\mathrm{Pb}$ collisions at the same multiplicity [14].

- Same factorization breakdown in $\mathrm{p}+\mathrm{A}$ and $\mathrm{A}+\mathrm{A}$. More differential tests along these lines are clearly useful in distinguishing between hydro models and with non-hydro models. At the present, hydro results for the breakdown in factorization of $v_{n}$ moments are sensitive to choices of initial conditions and $\eta / s$. Whether a hydro model "works" depends on all its parameters then being fixed by other pieces of data. 
- Similar HBT radii in $\mathrm{p}+\mathrm{A}$ and $\mathrm{A}+\mathrm{A}$. The correlation between HBT radii and flow is not straightforward. As a concrete example, the HBT radii in the highest multiplicity $p+p$ events are indistinguishable from the radii in $\mathrm{p}+\mathrm{A}$ collisions at the same multiplicity. However, the corresponding near side per trigger yield in $p+p$ is much smaller than that in $\mathrm{p}+\mathrm{A}$ collisions.

- Spectra better reproduced by event generators including hydro. The previous six criteria are experiment driven. While it is interesting that multiple scattering effects improve the agreement of event generators with the data, one should also note that a number of the initial state effects we have discussed here are also not included in the event generators.

One may ask further why there is no sign of mini-jet quenching in $\mathrm{p}+\mathrm{A}$ [15] ? In $\mathrm{A}+\mathrm{A}$ collisions, strong flow is accompanied by strong quenching. A related question is why $v_{2}$ is large in $\mathrm{p}+\mathrm{A}$ upto even $10 \mathrm{GeV}$ [16]. In $\mathrm{A}+\mathrm{A}$, this could be attributed to the strong path length dependence of partons traversing the hot matter. If energy loss is weak in $\mathrm{p}+\mathrm{A}$, the persistence of $v_{2}$ to higher $p_{T}$ is harder to understand. Why is the per trigger near side yield much smaller in $\mathrm{p}+\mathrm{p}$ than in $\mathrm{p}+\mathrm{A}$ for the same $N_{\mathrm{ch}}$ and HBT radii ? This last point is a challenge for otherwise very intriguing models that postulate conformal scaling to scale $\mathrm{p}+\mathrm{A}$ data on to $\mathrm{A}+\mathrm{A}$ data at the same multiplicity [17]. We note finally that while a number of hydrodynamic/transport models describe key aspects of the p+A data [18, 19, 20,21], they include very different assumptions about the initial state and $\eta / s$. Furthermore, collective flow models do not explain why the differential azimuthal anisotropies are largest at momenta of several $\mathrm{GeV}$, where hydrodynamics is least applicable.

\section{Conclusions}

The remarkable $\mathrm{p}+\mathrm{A}$ data has brought to the fore fundamental issues regarding how far one can extend the hydrodynamic paradigm to the smallest size systems, as well as the possiblity of extracting information about very rare multi-parton configurations in protons and nuclei. The challenge for theory is to come up with a consistent picture that addresses the many remaining puzzles outlined.

\section{Acknowledgements}

This research was supported by DOE Contract No. DE-AC02-98CH10886. I thank Kevin Dusling and Bjoern Schenke for their collaboration on the topics discussed here.

\section{References}

[1] H. Kowalski, L. Motyka and G. Watt, Phys. Rev. D 74, 074016 (2006); J. Breitweg et al., [ZEUS Collaboration] Phys. Lett. 487 (2000) 53 ; C. Adloff et al., [H1 Collaboration], Eur. Phys. J. C 24 (2002) 345.

[2] B. B. Abelev et al. [ALICE Collaboration], arXiv:1404.1194 [nucl-ex].

[3] A. Krasnitz, Y. Nara and R. Venugopalan, Nucl. Phys. A 727, 427 (2003)

[4] T. Lappi, Eur. Phys. J. C 55, 285 (2008).

[5] A. H. Rezaeian, M. Siddikov, M. Van de Klundert and R. Venugopalan, Phys. Rev. D 87, no. 3, 034002 (2013)

[6] K. Dusling, these proceeedings.

[7] K. Dusling and R. Venugopalan, Phys. Rev. D 87, no. 9, 094034 (2013).

[8] T. Lappi, S. Srednyak and R. Venugopalan, JHEP 1001, 066 (2010).

[9] M. Gyulassy, P. Levai, I. Vitev and T. Biro, arXiv:1405.7825 [hep-ph].

[10] Y. V. Kovchegov and D. E. Wertepny, Nucl. Phys. A 906, 50 (2013).

[11] A. Dumitru and A. V. Giannini, arXiv:1406.5781 [hep-ph].

[12] L. McLerran and V. V. Skokov, arXiv:1407.2651 [hep-ph].

[13] R. Granier de Cassagnac [CMS Collaboration], these proceedings.

[14] B. Schenke and R. Venugopalan, arXiv: 1405.3605 [nucl-th].

[15] Jan Fiete Grosse-Oetringhaus, [ALICE Collaboration], these proceedings.

[16] B. Cole, [ATLAS Collaboration], these proceeedings.

[17] G. Basar and D. Teaney, arXiv:1312.6770 [nucl-th].

[18] P. Bozek and W. Broniowski, Phys. Lett. B 718, 1557 (2013).

[19] K. Werner, M. Bleicher, B. Guiot, I. .Karpenko and T. Pierog, Phys. Rev. Lett. 112, 232301 (2014).

[20] I. Kozlov, M. Luzum, G. Denicol, S. Jeon and C. Gale, arXiv:1405.3976 [nucl-th].

[21] A. Bzdak and G. -L. Ma, arXiv:1406.2804 [hep-ph]. 